The Electronic Journal of Linear Algebra.

A publication of the International Linear Algebra Society.

Volume 7, pp. 152-173, September 2000.

ELA

ISSN 1081-3810.

\title{
DISTURBANCE DECOUPLING FOR DESCRIPTOR SYSTEMS BY MEASUREMENT FEEDBACK*
}

\author{
DELIN CHU ${ }^{\dagger}$ AND VOLKER MEHRMANN $\ddagger$
}

\begin{abstract}
In this paper, the disturbance decoupling problem for descriptor systems via feedbacks that are based on measurements is discussed. Necessary and sufficient conditions are derived for the existence of feedbacks so that the disturbances do not influence the input/output behavior. At the same time, the closed-loop system becomes regular, of index at most one, and possibly also stable. The construction is based on a transformation to condensed form under orthogonal equivalence transformations that can be directly implemented as a numerically stable method.
\end{abstract}

Key words. Disturbance decoupling, Descriptor systems, Orthogonal transformation, Condensed form

AMS subject classifications. 93B05, 93B40, 93B52, 65F35

1. Introduction. In this paper, we study the disturbance decoupling problem for descriptor systems by measurement feedback using methods that were developed recently in $[6,7,8]$. Consider a descriptor system of the form

$$
\begin{aligned}
E \dot{x} & =A x+B u+G d, \\
y & =C x, \\
z & =H x,
\end{aligned}
$$

where $x \in \mathbb{R}^{n}$ is the state, $u \in \mathbb{R}^{m}$ is the control input, $y \in \mathbb{R}^{p}$ is a measurement, $z \in \mathbb{R}^{l}$ is the output, and $d \in \mathbb{R}^{q}$ is a disturbance that may represent modeling or measuring errors, noise, higher-order terms in a linearization, or just an unknown input to the system. The system matrices satisfy $E, A \in \mathbb{R}^{n \times n}, B \in \mathbb{R}^{n \times m}, G \in \mathbb{R}^{n \times q}$, $C \in \mathbb{R}^{p \times n}$, and $H \in \mathbb{R}^{l \times n}$.

The topic of this paper is to design a feedback compensator for system (1), based on measurements, which has the form

$$
\begin{aligned}
E_{m} \dot{w} & =A_{m} w+L y \\
u & =M w+N y
\end{aligned}
$$

where $w \in \mathbb{R}^{k}$ is the state of the compensator and $k$ is the order of the compensator.

The closed-loop system obtained with this compensator is

$$
\begin{aligned}
\mathcal{E}\left[\begin{array}{c}
\dot{x} \\
\dot{w}
\end{array}\right] & =\mathcal{A}\left[\begin{array}{c}
x \\
w
\end{array}\right]+\left[\begin{array}{c}
G \\
0
\end{array}\right] d, \\
z & =\left[\begin{array}{ll}
H & 0
\end{array}\right]\left[\begin{array}{c}
x \\
w
\end{array}\right]
\end{aligned}
$$

${ }^{*}$ Received by the editors on 25 November 1999. Accepted for publication on 27 August 2000. Handling Editor: Daniel Hershkowitz

${ }^{\dagger}$ Department of Mathematics, National University of Singapore, 2 Science Drive 2, Singapore 117543 (matchudl@math.nus.edu.sg).

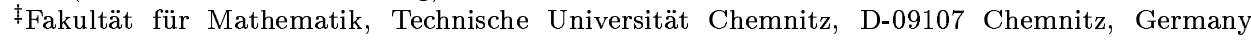
(mehrmann@mathematik.tu-chemnitz.de) 
with

$$
\mathcal{E}:=\left[\begin{array}{cc}
E & \\
& E_{m}
\end{array}\right], \quad \mathcal{A}:=\left[\begin{array}{cc}
A+B N C & B M \\
L C & A_{m}
\end{array}\right] .
$$

Apart from the requirement that the disturbances should not occur in the input/output relationship, i.e., that we have

$$
+\left[\begin{array}{ll}
H & 0
\end{array}\right]\left[\begin{array}{cc}
s E-A-B N C & -B M \\
-L C & s E_{m}-A_{m}
\end{array}\right]^{-1}\left[\begin{array}{c}
G \\
0
\end{array}\right]=0,
$$

further properties are desirable for the closed-loop system. First of all, the pencil of the closed-loop system $(3)$ should be regular, i.e., $\operatorname{det}(s \mathcal{E}-\mathcal{A})$ does not vanish identically for all $s$. This condition guarantees that the closed-loop system has a unique solution for all sufficiently smooth $d$ and appropriate initial conditions. Secondly, we would like the closed-loop system to have index at most one, i.e., all the Jordan blocks associated with infinite eigenvalues of the matrix pencil $\lambda \mathcal{E}-\mathcal{A}$ are of size at most one. This condition guarantees that no impulsive modes occur in the response of the system if piecewise-constant input functions are used; see [4, 5] for a dicussion of these issues. Finally, for obvious reasons, we would like the closed-loop system to be stable, i.e., all finite eigenvalues of the closed-loop system are in the open left half-plane.

Disturbance decoupling via state and output feedback has been discussed widely in the literature $[2,6,7,12,14]$. The use of measurement feedback, however, is more practical and has (to our knowledge) not been discussed so far. We will present necessary and sufficient conditions for the solution of these problems.

The basis for our results is a condensed form under orthogonal equivalence transformations, which is a variation of the generalized upper-triangular (GUPTRI) form for matrix pencils $[10,11]$. Numerical methods for the computation of this form are available in LAPACK [1]. The GUPTRI form displays all the invariants, in particular the left and right Kronecker indices, but it is not the complete canonical form.

LEMma 1.1. [10] Given a matrix pencil $(E, A), E, A \in \mathbb{R}^{n \times l}$, there exist orthogonal matrices $P \in \mathbb{R}^{n \times n}, Q \in \mathbb{R}^{l \times l}$ such that $(P E Q, P A Q)$ are in the following GUPTRI form:

$$
P(s E-A) Q=\begin{gathered}
l_{1} \\
n_{1} \\
n_{2} \\
n_{3} \\
n_{4}
\end{gathered}\left[\begin{array}{cccc}
s E_{11}-A_{11} & s E_{12}-A_{12} & s E_{13}-A_{13} & s E_{14}-A_{14} \\
0 & s E_{22}-A_{22} & s E_{23}-A_{23} & s E_{24}-A_{24} \\
0 & 0 & s E_{33}-A_{33} & s E_{34}-A_{34} \\
0 & 0 & 0 & s E_{44}-A_{44}
\end{array}\right]
$$

Here $n_{2}=l_{2}, n_{3}=l_{3}$, and $s E_{11}-A_{11}$ and $s E_{44}-A_{44}$ contain all left- and rightsingular Kronecker blocks of $s E-A$, respectively. Furthermore, $s E_{22}-A_{22}$ and $s E_{33}-A_{33}$ are regular and contain the regular finite and infinite structures of $s E-A$, respectively.

We use the following notation: $S_{\infty}(M)$ denotes a matrix with orthogonal columns spanning the right nullspace of a matrix $M, T_{\infty}(M)$ denotes a matrix with orthogonal 
columns spanning the right nullspace of a matrix $M^{T}, \operatorname{deg}(f(s))$ denotes the degree of the polynomial $f(s), \operatorname{rank}_{g}[\cdot](s)$ denotes the generic rank of a rational matrix function, $\overline{\mathbb{C}}^{+}$denotes the closed right half complex plane; and $\mathbb{C}^{-}$denotes the open left half complex plane.

Our necessary and sufficient conditions will depend on the quantities that can be read off from the condensed form that we determine in Section 2.

We will also formulate these conditions in terms of the original data of the system as well as geometric conditions related to dimensions of subspaces. This allows a coordinate-free statement of our results and control theoretic interpretations.

We recall three useful and well-known lemmas that we need in the proofs of the main theorems in the following section and that characterize separately the conditions that are needed to fulfill the requirements for the closed-loop system.

The first lemma characterizes when we can do the disturbance decoupling at all.

Lemma 1.2. [7] Consider matrices $E, A \in \mathbb{R}^{n \times n}, B \in \mathbb{R}^{n \times m}, C \in \mathbb{R}^{p \times n}$ and assume that $(E, A)$ is regular. Then $C(s E-A)^{-1} B=0$ if and only if

$$
\operatorname{rank}_{g}\left[\begin{array}{cc}
s E-A & B \\
C & 0
\end{array}\right]=n .
$$

Our second result is the characterization of when a system is regular and of index at most one.

Lemma 1.3. [4,9] Let $E, A \in \mathbb{R}^{n \times n}$. The following statements are equivalent:

(a) $(E, A)$ is regular and of index at most one.

(b) $\operatorname{rank}\left[\begin{array}{ll}E & A S_{\infty}(E)\end{array}\right]=n$.

(c) $\operatorname{rank}\left[\begin{array}{c}E \\ T_{\infty}(E)^{T} A\end{array}\right]=n$.

(d) $\operatorname{deg}(\operatorname{det}(s E-A))=\operatorname{rank}(E)$.

Finally, we have the characterization of when a system can be regularized to have index at most one and also when it can be made stable by feedback.

Lemma 1.4. $[4,9]$ Let $E, A \in \mathbb{R}^{n \times n}, B \in \mathbb{R}^{n \times m}$.

(a) There exists a matrix $F \in \mathbb{R}^{m \times n}$ such that $(E, A+B F)$ is regular if and only if

$$
\operatorname{rank}_{g}[s E-A \quad B]=n .
$$

(b) There exists a matrix $F \in \mathbb{R}^{m \times n}$ such that $(E, A+B F)$ is regular and stable if and only if

$$
\operatorname{rank}\left[\begin{array}{ll}
s E-A & B
\end{array}\right]=n \quad \forall s \in \overline{\mathbb{C}}^{+} .
$$

(c) There exists a matrix $F \in \mathbb{R}^{m \times n}$ such that $(E, A+B F)$ is regular and of index at most one if and only if (6) holds and

$$
\operatorname{rank}\left[\begin{array}{lll}
E & A S_{\infty}(E) & B
\end{array}\right]=n .
$$

(d) There exists a matrix $F \in \mathbb{R}^{m \times n}$ such that $(E, A+B F)$ is regular, stable, and of index at most one if and only if (7) and (8) hold. 
If the matrix $\left[\begin{array}{ll}s E-A & B\end{array}\right]$ has full rank for all $s \in \mathbb{C}$, then the associated system is called $R$-controllable and if $\left[\begin{array}{c}s E-A \\ C\end{array}\right]$ has full rank for all $s \in \mathbb{C}$ then the associated system is called $R$-observable; see $[9,4]$.

Condition (8) is often called controllability at infinity or impulse controllability, while the dual condition that

$$
\operatorname{rank}\left[\begin{array}{c}
T \\
T_{\infty}(E)^{T} A \\
B
\end{array}\right]
$$

is full is called observability at infinity or impulse observability; see [3, 4, 5, 9].

Note that the feedback matrices in Lemma 1.4 can be explicitly computed using numerically stable methods as suggested in $[4,15]$. Hence, in the following, if we make use of this lemma, it always implies that we have a numerical method that explicitly computes this feedback.

The paper is organized as follows. The basic tool that we use is a condensed form under orthogonal equivalence transformations. This condensed form is introduced in Section 2. In Section 3 we present our main results on the disturbance decoupling problem by measurement feedback. Some conclusions will be given in Section 4 .

2. A condensed form. In this section we will derive a condensed form under orthogonal equivalence transformations. The proof is constructive and can be directly implemented as a numerically stable algorithm. This condensed form will then be used in the following sections to prove our main results and will also provide explicit design methods for the desired feedbacks. The algorithm is based on row compressions and column compressions which can be obtained via singular value decompositions or other rank-revealing factorizations, e.g., $[1,13]$. Furthermore, the transformation of a matrix pencil to its GUPTRI form is used, which is implemented as the routine DGGBAK in LAPACK [1].

THEOREM 2.1. Consider a system of the form (1) with coefficient matrices $E$, $A, B, C, G$, and $H$. Then there exist orthogonal matrices $P, Q \in \mathbb{R}^{n \times n}$ such that

$$
\begin{aligned}
& \left.\begin{array}{ccccc}
\mu_{1} & \mu_{2} & \mu_{3} & \mu_{4} \\
\tau_{1} & \tau_{2} \\
& {[s E-A) Q=} & \mu_{3} \\
\tau_{4} & \tau_{5}-\Phi_{11} & s \Theta_{12}-\Phi_{12} & s \Theta_{13}-\Phi_{13} & s \Theta_{14}-\Phi_{14} \\
s \Theta_{21}-\Phi_{21} & s \Theta_{22}-\Phi_{22} & s \Theta_{23}-\Phi_{23} & s \Theta_{24}-\Phi_{24} \\
0 & -\Phi_{32} & s \Theta_{33}-\Phi_{33} & s \Theta_{34}-\Phi_{34} \\
0 & -\Phi_{42} & -\Phi_{43} & s \Theta_{44}-\Phi_{44} \\
0 & 0 & 0 & s \Theta_{54}-\Phi_{54}
\end{array}\right], \\
& P B=\begin{array}{c}
\tau_{1} \\
\tau_{2} \\
\mu_{3} \\
\tau_{4} \\
\tau_{5}
\end{array}\left[\begin{array}{c}
\Psi_{1} \\
\Psi_{2} \\
\Psi_{3} \\
\Psi_{4} \\
0
\end{array}\right], \quad P G=\mu_{3}\left[\begin{array}{c}
\tau_{1} \\
\tau_{4}
\end{array}\left[\begin{array}{c}
G_{1} \\
0 \\
0 \\
0 \\
0
\end{array}\right]\right.
\end{aligned}
$$




$$
C Q=\left[\begin{array}{llll}
\mu_{1} & \mu_{2} & \mu_{3} & \mu_{4} \\
0 & C_{2} & C_{3} & C_{4}
\end{array}\right], \quad H Q=\left[\begin{array}{cccc}
\mu_{1} & \mu_{2} & \mu_{3} & \mu_{4} \\
0 & 0 & 0 & \Pi_{4}
\end{array}\right]
$$

such that the following properties hold.

The matrices $G_{1}, \Psi_{4}$, and

$$
\left[\begin{array}{cccc}
\Theta_{21} & \Theta_{22} & \Theta_{23} & \Psi_{2} \\
0 & 0 & \Theta_{33} & \Psi_{3} \\
0 & 0 & 0 & \Psi_{4}
\end{array}\right]
$$

are of full row rank, $\Theta_{33}$ is nonsingular, $C_{2}$ is of full column rank, and, furthermore,

$$
\begin{aligned}
& \operatorname{rank}\left[\begin{array}{cccc}
s \Theta_{21}-\Phi_{21} & s \Theta_{22}-\Phi_{22} & s \Theta_{23}-\Phi_{23} & \Psi_{2} \\
0 & -\Phi_{32} & s \Theta_{33}-\Phi_{33} & \Psi_{3} \\
0 & -\Phi_{42} & -\Phi_{43} & \Psi_{4}
\end{array}\right]=\tau_{2}+\mu_{3}+\tau_{4} \quad \forall s \in \mathbb{C}, \\
& \operatorname{rank}\left[\begin{array}{cc}
-\Phi_{32} & s \Theta_{33}-\Phi_{33} \\
-\Phi_{42} & -\Phi_{43} \\
C_{2} & C_{3}
\end{array}\right]=\mu_{2}+\mu_{3} \quad \forall s \in \mathbb{C}, \\
& \operatorname{rank}_{g}\left(s \Theta_{21}-\Phi_{21}\right)=\tau_{2}, \\
& \operatorname{rank}_{g}\left[\begin{array}{c}
s \Theta_{54}-\Phi_{54} \\
\Pi_{4}
\end{array}\right]=\mu_{4} \text {. }
\end{aligned}
$$

Proof. We prove Theorem 2.1 constructively by the following algorithm.

\section{Algorithm}

Input: System matrices $E, A, B, G, C, H$ as in system (1).

Output: Orthogonal matrices $P, Q$, and the condensed form (9).

Step 1. Perform a row compression of [ $\left.\begin{array}{ll}B & G\end{array}\right]$ and a column compression of $H$ and then compute the GUPTRI form of $T_{\infty}^{T}\left(\left[\begin{array}{ll}B & G\end{array}\right]\right)(s E-A) S_{\infty}(H)$ to determine orthogonal matrices $P_{1}, Q_{1}$ such that

$$
\begin{aligned}
& P_{1}(s E-A) Q_{1}=\begin{array}{c}
\tau_{1} \\
\tau_{2}^{(1)} \\
\tau_{3}^{(1)} \\
\tau_{5}
\end{array}\left[\begin{array}{ccc}
s \Theta_{11}^{(1)}-\Phi_{4} & \mu_{41}^{(1)} & s \Theta_{14}-\Phi_{14} \\
s \Theta_{21}^{(1)}-\Phi_{21}^{(1)} & s \Theta_{22}^{(1)}-\Phi_{22}^{(1)} \\
s \Theta_{31}^{(1)}-\Phi_{31}^{(1)} & s \Theta_{32}^{(1)}-\Phi_{32}^{(1)} \\
0 & s \Theta_{54}-\Phi_{54}
\end{array}\right], \\
& n-\mu_{4} \quad \mu_{4} \\
& H Q_{1}=\left[\begin{array}{cc}
0 & \Pi_{4}
\end{array}\right] \text {, } \\
& P_{1} B=\begin{array}{l}
\tau_{1} \\
\tau_{2}^{(1)} \\
\tau_{3}^{(1)} \\
\tau_{5}
\end{array}\left[\begin{array}{c}
\Psi_{1} \\
\Psi_{2}^{(1)} \\
0 \\
0
\end{array}\right], \quad P_{1} G=\begin{array}{l}
\tau_{1} \\
\tau_{2}^{(1)} \\
\tau_{3}^{(1)} \\
\tau_{5}
\end{array}\left[\begin{array}{c}
G_{1} \\
0 \\
0 \\
0
\end{array}\right]
\end{aligned}
$$




\section{ELA}

where $\operatorname{rank}_{g}\left[\begin{array}{c}s \Theta_{54}-\Phi_{54} \\ \Pi_{4}\end{array}\right]=\mu_{4}$ (i.e., condition (11) holds), $\tau_{1}+\tau_{2}^{(1)}+\tau_{3}^{(1)}+\tau_{5}=n$, $\operatorname{rank}\left(G_{1}\right)=\tau_{1}, \operatorname{rank}\left(\Psi_{2}^{(1)}\right)=\tau_{2}^{(1)}, \operatorname{rank}\left(\Theta_{31}^{(1)}\right)=\tau_{3}^{(1)}$, and, furthermore,

$$
\operatorname{rank}\left(s \Theta_{31}^{(1)}-\Phi_{31}^{(1)}\right)=\tau_{3}^{(1)} \quad \forall s \in \mathbb{C} .
$$

Partition the matrix $C Q_{1}$ as

$$
C Q_{1}=\left[\begin{array}{cc}
n-\mu_{4} & \mu_{4} \\
C_{1}^{(1)} & C_{4}
\end{array}\right]
$$

Step 2. Perform a column compression of $C_{1}^{(1)}$ to get an orthogonal matrix $Q_{2}$ such that

$$
C_{1}^{(1)} Q_{2}=:\left[\begin{array}{cc}
t_{1} & n-\mu_{4}-t_{1} \\
0 & C_{2}^{(2)}
\end{array}\right]
$$

where $\operatorname{rank}\left(C_{2}^{(2)}\right)=n-\mu_{4}-t_{1}$. Set

$$
\begin{gathered}
{\left[\begin{array}{c}
s \Theta_{21}^{(1)}-\Phi_{21}^{(1)} \\
s \Theta_{31}^{(1)}-\Phi_{31}^{(1)}
\end{array}\right] Q_{2}=:\left[\begin{array}{cc}
t_{1} & n-\mu_{4}-t_{1} \\
s \Theta_{21}^{(2)}-\Phi_{21}^{(2)} & s \Theta_{22}^{(2)}-\Phi_{22}^{(2)}
\end{array}\right]} \\
t_{1} \\
\left(s \Theta_{11}^{(1)}-\Phi_{11}^{(1)}\right) Q_{2}=:\left[\begin{array}{cc}
n \Theta_{11}^{(2)}-\mu_{4}-t_{1} \\
(2) & s \Theta_{12}^{(2)}-\Phi_{12}^{(2)}
\end{array}\right] .
\end{gathered}
$$

Step 3. Compute the GUPTRI form of $s \Theta_{21}^{(2)}-\Phi_{21}^{(2)}$ to determine orthogonal matrices $P_{3}, Q_{3}$ such that

$$
P_{3}\left(s \Theta_{21}^{(2)}-\Phi_{21}^{(2)}\right) Q_{3}=: \tau_{2}^{(1)}+\tau_{3}^{(1)}-\tau_{2}\left[\begin{array}{cc}
\mu_{1} & t_{1}-\mu_{1} \\
\tau_{2} \Theta_{21}-\Phi_{21} & s \Theta_{22}^{(3)}-\Phi_{22}^{(3)} \\
0 & s \Theta_{32}^{(3)}-\Phi_{32}^{(3)}
\end{array}\right],
$$

where $\operatorname{rank}\left(\Theta_{32}^{(3)}\right)=t_{1}-\mu_{1}, \operatorname{rank}_{g}\left(s \Theta_{21}-\Phi_{21}\right)=\tau_{2}$ (i.e., condition (10) holds), and

$$
\operatorname{rank}\left(s \Theta_{32}^{(3)}-\Phi_{32}^{(3)}\right)=t_{1}-\mu_{1} \quad \forall s \in \mathbb{C} .
$$

Set

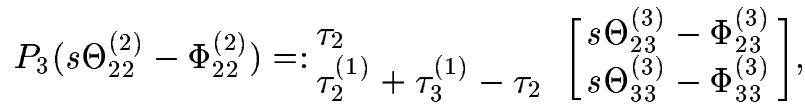

$$
\begin{aligned}
& \left(s \Theta_{11}^{(2)}-\Phi_{11}^{(2)}\right) Q_{3}=:\left[\begin{array}{cc}
\mu_{1} & t_{1}-\mu_{1} \\
s \Theta_{11}-\Phi_{11} & s \Theta_{12}^{(3)}-\Phi_{12}^{(3)}
\end{array}\right],
\end{aligned}
$$




\section{ELA}

and

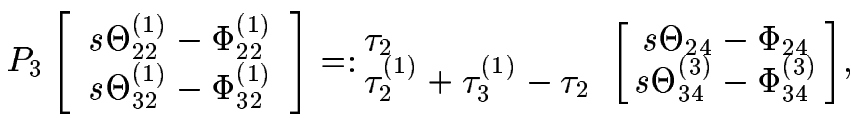

$$
\begin{aligned}
& P_{3}\left[\begin{array}{c}
\Psi_{2}^{(1)} \\
0
\end{array}\right]=:{ }_{\tau_{2}^{(1)}}^{\tau_{2}}+\tau_{3}^{(1)}-\tau_{2}\left[\begin{array}{c}
\Psi_{2} \\
\Psi_{3}^{(3)}
\end{array}\right] .
\end{aligned}
$$

Step 4. Perform a simultaneous row and column compression of $\left[\begin{array}{cc}\Theta_{32}^{(3)} & \Theta_{33}^{(3)}\end{array}\right]$ to get orthogonal matrices $P_{4}$ and $Q_{4}$ such that

$$
P_{4}\left[s \Theta_{32}^{(3)}-\Phi_{32}^{(3)} \quad s \Theta_{33}^{(3)}-\Phi_{33}^{(3)}\right] Q_{4}=: \begin{array}{cc}
\mu_{2} & \mu_{3} \\
\tau_{4} & {\left[\begin{array}{cc}
-\Phi_{32} & s \Theta_{33}-\Phi_{33} \\
-\Phi_{42} & -\Phi_{43}
\end{array}\right],}
\end{array}
$$

where $\Theta_{33}$ is nonsingular and

$\mu_{2}=\left(n-\mu_{4}-t_{1}\right)+\left(t_{1}-\mu_{1}\right)-\mu_{3}=n-\mu_{1}-\mu_{3}-\mu_{4}, \tau_{4}=\tau_{2}^{(1)}+\tau_{3}^{(1)}-\tau_{2}-\mu_{3}$.

Set

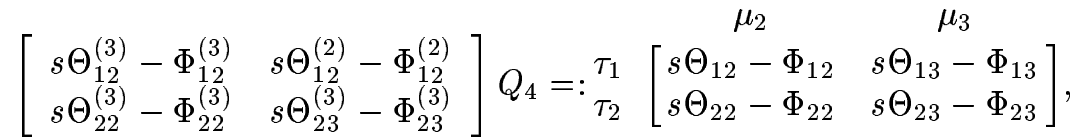

$$
\begin{aligned}
& {\left[\begin{array}{ll}
0 & C_{2}^{(2)}
\end{array}\right] Q_{4}=:\left[\begin{array}{ll}
\mu_{2} & \mu_{3} \\
C_{2} & C_{3}
\end{array}\right],} \\
& P_{4}\left(s \Theta_{34}^{(3)}-\Phi_{34}^{(3)}\right)=:{ }_{\tau_{4}}^{\mu_{3}}\left[\begin{array}{l}
s \Theta_{34}-\Phi_{34} \\
s \Theta_{44}-\Phi_{44}
\end{array}\right] \\
& P_{4} \Psi_{3}^{(3)}=:{ }_{\tau_{4}}^{\mu_{3}}\left[\begin{array}{l}
\Psi_{3} \\
\Psi_{4}
\end{array}\right],
\end{aligned}
$$

and

$$
\begin{aligned}
& P:=\left[\begin{array}{lll}
I_{\tau_{1}+\tau_{2}} & & \\
& P_{4} & \\
& & I_{\tau_{5}}
\end{array}\right]\left[\begin{array}{lll}
I_{\tau_{1}} & & \\
& P_{3} & \\
& & I_{\tau_{5}}
\end{array}\right] P_{1}, \\
& Q:=Q_{1}\left[\begin{array}{ll}
Q_{2} & \\
& I_{\mu_{4}}
\end{array}\right]\left[\begin{array}{lll}
Q_{3} & \\
& I_{t_{3}+\mu_{4}}
\end{array}\right]\left[\begin{array}{lll}
I_{\mu_{1}} & & \\
& Q_{4} & \\
& & I_{\mu_{4}}
\end{array}\right] .
\end{aligned}
$$


Note that

$$
\begin{aligned}
& \operatorname{rank}\left[\begin{array}{cccc}
s \Theta_{21}-\Phi_{21} & s \Theta_{22}-\Phi_{22} & s \Theta_{23}-\Phi_{23} & \Psi_{2} \\
0 & -\Phi_{32} & s \Theta_{33}-\Phi_{33} & \Psi_{3} \\
0 & -\Phi_{42} & -\Phi_{43} & \Psi_{4}
\end{array}\right] \\
= & \operatorname{rank}\left[\begin{array}{cc}
s \Theta_{21}^{(1)}-\Phi_{21}^{(1)} & \Psi_{2}^{(1)} \\
s \Theta_{31}^{(1)}-\Phi_{31}^{(1)} & 0
\end{array}\right]=\tau_{2}^{(1)}+\tau_{3}^{(1)}=\tau_{2}+\mu_{3}+\tau_{4} \quad \forall s \in \mathbb{C}
\end{aligned}
$$

and

$$
\begin{aligned}
& \operatorname{rank}\left[\begin{array}{cc}
-\Phi_{32} & s \Theta_{33}-\Phi_{33} \\
-\Phi_{42} & -\Phi_{43} \\
C_{2} & C_{3}
\end{array}\right]=\operatorname{rank}\left[\begin{array}{cc}
s \Theta_{32}^{(3)}-\Phi_{32}^{(3)} & s \Theta_{33}^{(3)}-\Phi_{33}^{(3)} \\
0 & C_{2}^{(2)}
\end{array}\right] \\
= & \left(n-\mu_{4}-t_{1}\right)+\left(t_{1}-\mu_{1}\right)=n-\mu_{1}-\mu_{4}=\mu_{2}+\mu_{3} \quad \forall s \in \mathbb{C} .
\end{aligned}
$$

Hence, the rank conditions hold and $P E Q, P A Q, P B, P G, C Q$, and $H Q$ are in the condensed form (9).

In the following section, we will use condensed form (9) to derive solvability conditions for the disturbance decoupling problem by measurement feedback. In order to formulate our solvability conditions in terms of the original data of the system (1), we need some notation.

Definition 2.2. [10] Given a matrix pencil $(E, A), E, A \in \mathbb{R}^{n \times l}$, and orthogonal matrices $P, Q$ such that $P(s E-A) Q$ is of the form (5), then

1. The minimal left-reducing subspace $V_{m-l}[E, A]$ of $(E, A)$ is the space spanned by the leading $n_{1}$ columns of $P^{T}$.

2. The minimal right-reducing subspace $V_{m-r}[E, A]$ of $(E, A)$ is the space spanned by the leading $l_{1}$ columns of $Q$.

3. The left-reducing subspace corresponding to the finite spectrum of $(E, A)$, denoted by $V_{f-l}[E, A]$, is the space spanned by the leading $n_{1}+n_{2}$ columns of $P^{T}$.

4. The right-reducing subspace corresponding to the finite spectrum of $(E, A)$, denoted by $V_{f-r}[E, A]$, is the space spanned by the leading $l_{1}+l_{2}$ columns of $Q$.

Introduce, furthermore, the following spaces:

$$
\begin{aligned}
& W:=T_{\infty}\left[\begin{array}{cc}
B & G \\
0 & 0
\end{array}\right], \quad \Lambda_{r}:=V_{m-r}\left[W^{T}\left[\begin{array}{c}
E \\
0
\end{array}\right], W^{T}\left[\begin{array}{c}
A \\
H
\end{array}\right]\right], \\
& \Lambda_{l}:=V_{m-l}\left[W^{T}\left[\begin{array}{c}
E \\
0
\end{array}\right], W^{T}\left[\begin{array}{c}
A \\
H
\end{array}\right]\right], \Lambda_{t}:=\left[\begin{array}{ll}
W^{\perp} & W
\end{array}\right]\left[\begin{array}{cc}
I & 0 \\
0 & \Lambda_{l}
\end{array}\right], \\
& \Lambda_{1}:=\Lambda_{t}^{T}\left[\begin{array}{c}
E \\
0
\end{array}\right] \Lambda_{r}, \quad \Lambda_{2}:=\Lambda_{t}^{T}\left[\begin{array}{c}
A \\
H
\end{array}\right] \Lambda_{r}, \\
& \Lambda_{3}:=\Lambda_{t}^{T}\left[\begin{array}{c}
B \\
0
\end{array}\right], \quad \Lambda_{4}:=\Lambda_{t}^{T}\left[\begin{array}{c}
G \\
0
\end{array}\right] .
\end{aligned}
$$


Moreover, let

$$
\begin{aligned}
\Lambda_{5} & :=T_{\infty}^{T}(G)\left[\begin{array}{c}
E \\
0
\end{array}\right], \quad \Lambda_{6}=T_{\infty}^{T}(G)\left[\begin{array}{l}
A \\
C
\end{array}\right], \\
\Pi & :=V_{f-l}\left[\Lambda_{5} \Lambda_{r}, \Lambda_{6} \Lambda_{r}\right], \\
\Xi_{1} & :=\left(\Pi^{\perp}\right)^{T} \Lambda_{5}, \quad \Xi_{2}:=\left(\Pi^{\perp}\right)^{T} \Lambda_{6}, \quad \Xi_{3}=\left(\Pi^{\perp}\right)^{T} \Lambda_{5} \Lambda_{r} .
\end{aligned}
$$

Here ${ }^{\perp}$ denotes the orthogonal complement of a space.

Using this notation we can read off the following dimensions of subspaces from the condensed form (1).

Corollary 2.3. Consider a system of the form (1) and let $P$ and $Q$ be orthogonal matrices $P$ such that $P E Q, P A Q, P B, P G, C Q$, and $H Q$ are in the condensed form (9). Introduce submatrices in the condensed form (9) as follows:

$$
\begin{aligned}
{\left[s \tilde{E}_{1}-\tilde{A}_{1} \mid \tilde{B}\right] } & =\left[\begin{array}{ccc|c}
s \Theta_{11}-\Phi_{11} & s \Theta_{12}-\Phi_{12} & s \Theta_{13}-\Phi_{13} & \Psi_{1} \\
s \Theta_{21}-\Phi_{21} & s \Theta_{22}-\Phi_{22} & s \Theta_{23}-\Phi_{23} & \Psi_{2} \\
0 & -\Phi_{32} & s \Theta_{33}-\Phi_{33} & \Psi_{3} \\
0 & -\Phi_{42} & -\Phi_{43} & \Psi_{4}
\end{array}\right] \\
{\left[\begin{array}{c}
s \tilde{E}_{2}-\tilde{A}_{2} \\
\hline \tilde{C}
\end{array}\right] } & =\left[\begin{array}{ccc}
-\Phi_{32} & s \Theta_{33}-\Phi_{33} & s \Theta_{34}-\Phi_{34} \\
-\Phi_{42} & -\Phi_{43} & s \Theta_{44}-\Phi_{44} \\
0 & 0 & s \Theta_{54}-\Phi_{54} \\
\hline C_{2} & C_{3} & C_{4}
\end{array}\right]
\end{aligned}
$$

Then,

$$
\begin{aligned}
& \operatorname{rank}\left[s \Lambda_{1}-\Lambda_{2}\right.\left.\Lambda_{3}\right] \\
& \operatorname{rank}\left(s \Xi_{1}-\Xi_{2}\right)=\operatorname{rank}\left[\begin{array}{cc}
s \tilde{E}_{1}-\tilde{A}_{1} & \tilde{B}
\end{array}\right] \quad \forall s \in \mathbb{C}, \\
& \mu_{1}+\mu_{2}+\mu_{3}-\tilde{A}_{2}=\operatorname{rank}\left(\Lambda_{r}\right), \quad \forall s \in \mathbb{C}, \\
& \tau_{1}+\tau_{2}+\mu_{3}+\tau_{4}=\operatorname{rank}\left(\Lambda_{t}\right), \\
& \mu_{2}+\mu_{3}+\mu_{4}=n-\operatorname{rank}\left(V_{f-r}\left[\Lambda_{5} \Lambda_{r}, \Lambda_{6} \Lambda_{r}\right]\right), \\
& \tau_{1}=\operatorname{rank}(G), \quad \tau_{2}=\operatorname{rank}(\Pi), \quad \mu_{3}=\operatorname{rank}\left(\Xi_{3}\right) .
\end{aligned}
$$

Using Corollary 2.3, we can relate subspaces in terms of the original data to subspaces in terms of the transformed data in the condensed form (9). We will make use of this in our main theorems in the next section.

3. Main results. In this section, we present our two main theorems. The first theorem gives necessary and the second sufficient conditions for the existence of feedbacks that make a system regular, of index at most one, and stable using measurement feedback.

THEOREM 3.1. Consider a system of the form (1) and assume that orthogonal matrices $P$ are given such that $P E Q, P A Q, P B, P G, C Q$, and $H Q$ are in the condensed form (9). Let $\tilde{E}_{1}, \tilde{E}_{2}, \tilde{A}_{1}, \tilde{A}_{2}, \tilde{B}, \tilde{C}, \Lambda_{i}, i=1, \ldots, 6, \Lambda_{r}, \Lambda_{t}, \Pi, \Xi_{j}$, $j=1,2,3$, be as in Corollary 2.3 . 
(a) If there exists a compensator of the form (2), with $\left(E_{m}, A_{m}\right)$ regular and of index at most one, such that the pencil of the closed-loop system (3) is regular and the disturbance decoupling relation (4) holds, then the following conditions hold:

$$
\begin{aligned}
\operatorname{rank}_{g}\left[\begin{array}{cc}
s E-A & B
\end{array}\right] & =n, \\
\operatorname{rank}_{g}\left[\begin{array}{c}
s E-A \\
C
\end{array}\right] & =n, \\
\operatorname{rank}(G)+\operatorname{rank}(\Pi)+\operatorname{rank}\left(\Xi_{3}\right) & \leq \operatorname{rank}\left(\Lambda_{r}\right), \\
\operatorname{rank}_{g}\left[s \Lambda_{1}-\Lambda_{2} \Lambda_{3}\right] & =\operatorname{rank}\left(\Lambda_{t}\right), \\
\operatorname{rank}_{g}\left(s \Xi_{1}-\Xi_{2}\right) & =n-\operatorname{rank}\left(V_{f-r}\left[\Lambda_{5} \Lambda_{r}, \Lambda_{6} \Lambda_{r}\right]\right) .
\end{aligned}
$$

(b) If the closed-loop system (3) is regular, stable, and satisfies (4), then the following conditions hold:

$$
\begin{aligned}
& \operatorname{rank}\left[\begin{array}{cc}
s E-A & B
\end{array}\right]=n \quad \forall s \in \overline{\mathbb{C}}^{+}, \\
& \operatorname{rank}\left[\begin{array}{c}
s E-A \\
C
\end{array}\right]=n \quad \forall s \in \overline{\mathbb{C}}^{+}, \\
& \text {(19) } \operatorname{rank}\left[\begin{array}{cc}
s \Lambda_{1}-\Lambda_{2} & \Lambda_{3}
\end{array}\right]=\operatorname{rank}\left(\Lambda_{t}\right) \quad \forall s \in \overline{\mathbb{C}}^{+} \text {, } \\
& \operatorname{rank}\left(s \Xi_{1}-\Xi_{2}\right)=n-\operatorname{rank}\left(V_{f-r}\left[\Lambda_{5} \Lambda_{r}, \Lambda_{6} \Lambda_{r}\right]\right) \quad \forall s \in \overline{\mathbb{C}}^{+} .
\end{aligned}
$$

(c) If the closed-loop system (3) is regular and of index at most one and satisfies (4), then the following conditions hold:

$$
\begin{aligned}
& \operatorname{rank}\left[\begin{array}{lll}
E & A S_{\infty}(E) & B
\end{array}\right]=n, \\
& \operatorname{rank}\left[\begin{array}{c}
E \\
T_{\infty}^{T}(E) A \\
C
\end{array}\right]=n, \\
& \operatorname{rank}\left(\tilde{E}_{1}\right)+\operatorname{rank}\left(\tilde{E}_{2}\right)=\operatorname{rank}(E)+\operatorname{rank}\left(\Xi_{3}\right), \\
& \operatorname{rank}\left[\begin{array}{ccc}
\Lambda_{1} & \Lambda_{2} S_{\infty}\left(\Lambda_{1}\right) & \Lambda_{3}
\end{array}\right]=\operatorname{rank}\left(\Lambda_{t}\right), \\
& \operatorname{rank}\left[\begin{array}{c}
\Xi_{1} \\
T_{\infty}^{T}\left(\Xi_{1}\right) \Xi_{2}
\end{array}\right]=n-\operatorname{rank}\left(V_{f-r}\left[\Lambda_{5} \Lambda_{r}, \Lambda_{6} \Lambda_{r}\right]\right) .
\end{aligned}
$$

(d) If the closed-loop system (3) is regular, of index at most one, stable, and satisfies (4), then conditions (17)-(25) hold.

Proof. Using Corollary 2.3, we can reformulate the conditions (14), (15), (16), (19), (20), (23), (24), and (25) as

$$
\begin{aligned}
& \tau_{1}+\tau_{2} \leq \mu_{1}+\mu_{2}, \\
& \operatorname{rank}_{g}\left[\begin{array}{cc}
s \tilde{E}_{1}-\tilde{A}_{1} & \tilde{B}
\end{array}\right]=\tau_{1}+\tau_{2}+\mu_{3}+\tau_{4} \text {, } \\
& \operatorname{rank}_{g}\left[\begin{array}{c}
s \tilde{E}_{2}-\tilde{A}_{2} \\
\tilde{C}
\end{array}\right]=\mu_{2}+\mu_{3}+\mu_{4},
\end{aligned}
$$




$$
\operatorname{rank}\left[\begin{array}{cc}
s \tilde{E}_{1}-\tilde{A}_{1} & \tilde{B}
\end{array}\right]=\tau_{1}+\tau_{2}+\mu_{3}+\tau_{4} \quad \forall s \in \overline{\mathbb{C}}^{+},
$$

$$
\operatorname{rank}\left[\begin{array}{c}
s \tilde{E}_{2}-\tilde{A}_{2} \\
\tilde{C}
\end{array}\right]=\mu_{2}+\mu_{3}+\mu_{4} \quad \forall s \in \overline{\mathbb{C}}^{+},
$$

$$
\begin{aligned}
& \operatorname{rank}\left[\begin{array}{cc}
\Theta_{11} & \Theta_{12} \\
\Theta_{21} & \Theta_{22}
\end{array}\right]+\operatorname{rank}\left[\begin{array}{c}
\Theta_{44} \\
\Theta_{54}
\end{array}\right]=\operatorname{rank}(E)-\mu_{3}, \\
& \operatorname{rank}\left[\begin{array}{ccc}
\tilde{E}_{1} & \tilde{A}_{1} S_{\infty}\left(\tilde{E}_{1}\right) & \tilde{B}
\end{array}\right]=\tau_{1}+\tau_{2}+\mu_{3}+\tau_{4}, \\
& \operatorname{rank}\left[\begin{array}{c}
\tilde{E}_{2} \\
T_{\infty}^{T}\left(\tilde{E}_{2}\right) \tilde{A}_{2} \\
\tilde{C}
\end{array}\right]=\mu_{2}+\mu_{3}+\mu_{4},
\end{aligned}
$$

respectively. Recall that

$$
\mathcal{E}=\left[\begin{array}{ll}
E & \\
& E_{m}
\end{array}\right], \quad \mathcal{A}=\left[\begin{array}{ll}
A & \\
& 0
\end{array}\right]+\left[\begin{array}{cc}
B & \\
& I_{k}
\end{array}\right]\left[\begin{array}{cc}
N & M \\
L & A_{m}
\end{array}\right]\left[\begin{array}{ll}
C & \\
& I_{k}
\end{array}\right] .
$$

(a) Conditions (12) and (13) follow directly from Lemma 1.4 (a).

Since $\Theta_{33}$ is nonsingular and $\left(E_{m}, A_{m}\right)$ is regular and of index at most one, it follows that the pencil

$$
\left(\left[\begin{array}{cc}
\Theta_{33} & \\
& E_{m}
\end{array}\right],\left[\begin{array}{cc}
\Phi_{33}+\Psi_{3} N C_{3} & \Psi_{3} M \\
L C_{3} & A_{m}
\end{array}\right]\right)
$$

is regular and of index at most one. By Lemma 1.2, we have

$$
\begin{aligned}
& n+k=\operatorname{rank}_{g}\left[\begin{array}{ccc}
s E-A-B N C & -B M & G \\
-L C & s E_{m}-A_{m} & 0 \\
H & 0 & 0
\end{array}\right] \\
= & \tau_{1}+\tau_{2}+\mu_{4}+\operatorname{rank}_{g}\left[\begin{array}{ccc}
-\Phi_{32}-\Psi_{3} N C_{2} & s \Theta_{33}-\Phi_{33}-\Psi_{3} N C_{3} & -\Psi_{3} M \\
-\Phi_{42}-\Psi_{4} N C_{2} & -\Phi_{43}-\Psi_{4} N C_{3} & -\Psi_{4} M \\
-L C_{2} & -L C_{3} & s E_{m}-A_{m}
\end{array}\right] \\
\geq & \tau_{1}+\tau_{2}+\mu_{4}+\operatorname{rank}_{g}\left[\begin{array}{cc}
s \Theta_{33}-\Phi_{33}-\Psi_{3} N C_{3} & -\Psi_{3} M \\
-L C_{3} & s E_{m}-A_{m}
\end{array}\right] \\
= & \tau_{1}+\tau_{2}+\mu_{4}+\mu_{3}+k \\
= & \tau_{1}+\tau_{2}+\left(n-\mu_{1}-\mu_{2}\right)+k .
\end{aligned}
$$

Hence, we have (26).

Consider the GUPTRI form

$$
\begin{aligned}
& U\left[\begin{array}{ccc}
-\Phi_{32}-\Psi_{3} N C_{2} & s \Theta_{33}-\Phi_{33}-\Psi_{3} N C_{3} & -\Psi_{3} M \\
-\Phi_{42}-\Psi_{4} N C_{2} & -\Phi_{43}-\Psi_{4} N C_{3} & -\Psi_{4} M \\
-L C_{2} & -L C_{3} & s E_{m}-A_{m}
\end{array}\right] V \\
& v \quad\left(\mu_{2}+\mu_{3}+k\right)-v \\
& =\begin{array}{l}
w \\
\left(\mu_{3}+\tau_{4}+k\right)-w
\end{array}\left[\begin{array}{cc}
s \tilde{\Theta}_{32}-\tilde{\Phi}_{32} & s \tilde{\Theta}_{33}-\tilde{\Phi}_{33} \\
0 & s \tilde{\Theta}_{43}-\tilde{\Phi}_{43}
\end{array}\right],
\end{aligned}
$$




\section{ELA}

with $U, V$ orthogonal, $\tilde{\Theta}_{32}$ of full row rank, $\operatorname{rank}_{g}\left(s \tilde{\Theta}_{43}-\tilde{\Phi}_{43}\right)=\left(\mu_{2}+\mu_{3}+k\right)-v$, and $\operatorname{rank}\left(s \tilde{\Theta}_{32}-\tilde{\Phi}_{32}\right)=w$ for all $s \in \mathbb{C}$. Set

$$
\begin{aligned}
& {\left[\begin{array}{ll}
\Psi_{1} & 0
\end{array}\right]=: \tilde{\Psi}_{1}, \quad\left[\begin{array}{ll}
\Psi_{2} & 0
\end{array}\right]=: \tilde{\Psi}_{2}, \quad\left[\begin{array}{c}
C_{4} \\
0
\end{array}\right]=: \tilde{C}_{4},} \\
& U\left[\begin{array}{c}
s \Theta_{34}-\Phi_{34}-\Psi_{3} N C_{4} \\
s \Theta_{44}-\Phi_{44}-\Psi_{4} N C_{4} \\
L C_{4}
\end{array}\right]=:\left[\begin{array}{c}
s \tilde{\Theta}_{34}-\tilde{\Phi}_{34} \\
s \tilde{\Theta}_{44}-\tilde{\Phi}_{34}
\end{array}\right], U\left[\begin{array}{cc}
\Psi_{3} & 0 \\
\Psi_{4} & 0 \\
0 & I
\end{array}\right]=:\left[\begin{array}{c}
\tilde{\Psi}_{3} \\
\tilde{\Psi}_{4}
\end{array}\right], \\
& {\left[\begin{array}{ccc}
s \Theta_{12}-\Phi_{12}-\Psi_{1} N C_{2} & s \Theta_{13}-\Phi_{13}-\Psi_{1} N C_{3} & \Psi_{1} M \\
s \Theta_{22}-\Phi_{22}-\Psi_{2} N C_{2} & s \Theta_{23}-\Phi_{23}-\Psi_{2} N C_{3} & \Psi_{2} M
\end{array}\right] V} \\
& =:\left[\begin{array}{cc}
s \tilde{\Theta}_{12}-\tilde{\Phi}_{12} & s \tilde{\Theta}_{13}-\tilde{\Phi}_{13} \\
s \tilde{\Theta}_{22}-\tilde{\Phi}_{22} & s \tilde{\Theta}_{23}-\tilde{\Phi}_{23}
\end{array}\right], \\
& {\left[\begin{array}{ccc}
C_{2} & C_{3} & 0 \\
0 & 0 & I
\end{array}\right] V=:\left[\begin{array}{cc}
\tilde{C}_{2} & \tilde{C}_{3}
\end{array}\right] \text {. }}
\end{aligned}
$$

Then, by Lemma 1.2, we have

$$
\begin{aligned}
\sum_{i=1}^{4} \mu_{i}+k & =n+k \\
& =\tau_{1}+\tau_{2}+\mu_{4}+w+\left[\left(\mu_{2}+\mu_{3}+k\right)-v\right],
\end{aligned}
$$

and then

$$
\mu_{1}+v=\tau_{1}+\tau_{2}+w
$$

Moreover, we have that

$$
\left[\begin{array}{cc}
s \Theta_{11}-\Phi_{11} & s \tilde{\Theta}_{12}-\tilde{\Phi}_{12} \\
s \Theta_{21}-\Phi_{21} & s \tilde{\Theta}_{22}-\tilde{\Phi}_{22} \\
0 & s \tilde{\Theta}_{32}-\tilde{\Phi}_{32}
\end{array}\right]
$$

is square. Since

$$
\left[\begin{array}{cc}
A+B N C & B M \\
L C & K
\end{array}\right]
$$

is regular, we then have the following identities for at least one $s \in \mathbb{C}$ :

$$
\begin{aligned}
\operatorname{rank}\left[\begin{array}{cc}
s \Theta_{11}-\Phi_{11} & s \tilde{\Theta}_{12}-\tilde{\Phi}_{12} \\
s \Theta_{21}-\Phi_{21} & s \tilde{\Theta}_{22}-\tilde{\Phi}_{22} \\
0 & s \tilde{\Theta}_{32}-\tilde{\Phi}_{32}
\end{array}\right] & =\tau_{1}+\tau_{2}+w \\
\operatorname{rank}\left[\begin{array}{cc}
s \tilde{\Theta}_{43}-\tilde{\Phi}_{43} & s \tilde{\Theta}_{44}-\tilde{\Phi}_{44} \\
0 & s \Theta_{54}-\Phi_{54}
\end{array}\right] & =\mu_{4}+\left(\mu_{2}+\mu_{3}+k\right)-v .
\end{aligned}
$$


On the other hand, from Theorem 2.1, we have that for all $s \in \mathbb{C}$,

$$
\begin{aligned}
\operatorname{rank}\left[s \tilde{\Theta}_{43}-\tilde{\Phi}_{43}\right. & \left.\tilde{\Psi}_{4}\right]=\left(\mu_{3}+\tau_{4}+k\right)-w, \\
{\left[\begin{array}{c}
s \tilde{\Theta}_{32}-\tilde{\Phi}_{32} \\
\tilde{C}_{2}
\end{array}\right] } & =v .
\end{aligned}
$$

Therefore, for at least one $s \in \mathbb{C}$, we have

$(36)$

$$
\begin{aligned}
& \operatorname{rank}\left[\begin{array}{ccccc}
s \Theta_{11}-\Phi_{11} & s \Theta_{12}-\Phi_{12} & s \Theta_{13}-\Phi_{13} & \Psi_{1} & 0 \\
s \Theta_{21}-\Phi_{21} & s \Theta_{22}-\Phi_{22} & s \Theta_{23}-\Phi_{23} & \Psi_{2} & 0 \\
0 & -\Phi_{32} & s \Theta_{33}-\Phi_{33} & \Psi_{3} & 0 \\
0 & -\Phi_{42} & -\Phi_{43} & \Psi_{4} & 0 \\
0 & 0 & 0 & 0 & I_{k}
\end{array}\right] \\
= & \operatorname{rank}\left[\begin{array}{cccc}
s \Theta_{11}-\Phi_{11} & s \tilde{\Theta}_{12}-\tilde{\Phi}_{12} & s \tilde{\Theta}_{13}-\tilde{\Phi}_{13} & \tilde{\Psi}_{1} \\
s \Theta_{21}-\Phi_{21} & s \tilde{\Theta}_{22}-\tilde{\Phi}_{22} & s \tilde{\Theta}_{23}-\tilde{\Phi}_{23} & \tilde{\Psi}_{2} \\
0 & s \tilde{\Theta}_{32}-\tilde{\Phi}_{32} & s \tilde{\Theta}_{33}-\tilde{\Phi}_{33} & \tilde{\Psi}_{3} \\
0 & 0 & s \tilde{\Theta}_{43}-\tilde{\Phi}_{43} & \tilde{\Psi}_{4}
\end{array}\right] \\
= & \tau_{1}+\tau_{2}+w+\left(\mu_{3}+\tau_{4}+k\right)-w \\
= & \tau_{1}+\tau_{2}+\mu_{3}+\tau_{4}+k
\end{aligned}
$$

and

$$
\begin{aligned}
& \operatorname{rank}\left[\begin{array}{cccc}
-\Phi_{32} & s \Theta_{33}-\Phi_{33} & s \Theta_{34}-\Phi_{34} & 0 \\
-\Phi_{42} & -\Phi_{43} & s \Theta_{44}-\Phi_{44} & 0 \\
0 & 0 & s \Theta_{54}-\Phi_{54} & 0 \\
C_{2} & C_{3} & C_{4} & 0 \\
0 & 0 & 0 & I_{k}
\end{array}\right] \\
= & \operatorname{rank}\left[\begin{array}{ccc}
s \tilde{\Theta}_{32}-\tilde{\Phi}_{32} & s \tilde{\Theta}_{33}-\tilde{\Phi}_{33} & s \tilde{\Theta}_{34}-\tilde{\Phi}_{34} \\
0 & s \tilde{\Theta}_{43}-\tilde{\Phi}_{43} & s \tilde{\Theta}_{44}-\tilde{\Phi}_{44} \\
0 & 0 & s \Theta_{54}-\Phi_{54} \\
\tilde{C}_{2} & \tilde{C}_{3} & \tilde{C}_{4}
\end{array}\right] \\
= & v+\left[\left(\mu_{2}+\mu_{3}+k\right)-v\right]+\mu_{4} \\
= & \sum_{i=2}^{4} \mu_{i}+k,
\end{aligned}
$$

and conditions (27) and (28) follow.

(b) If the pencil $(\mathcal{E}, \mathcal{A})$ is stable, then conditions (17) and (18) follow directly from Lemma 1.4 (b). Moreover, (34) and (35) hold for all $s \in \overline{\mathbb{C}}^{+}$and hence (36) and (37) hold for all $s \in \overline{\mathbb{C}}^{+}$. This implies that (29) and (30) hold.

(c) If the pencil $(\mathcal{E}, \mathcal{A})$ is of index at most one, then conditions (21) and (22) follow directly from Lemma 1.4 (c). In order to prove conditions (32) and (33), we introduce the submatrices

$$
s \hat{\Theta}_{1}-\hat{\Phi}_{1}:=\left[\begin{array}{cc}
s \Theta_{11}-\Phi_{11} & s \tilde{\Theta}_{12}-\tilde{\Phi}_{12} \\
s \Theta_{21}-\Phi_{21} & s \tilde{\Theta}_{22}-\tilde{\Phi}_{22} \\
0 & s \tilde{\Theta}_{32}-\tilde{\Phi}_{32}
\end{array}\right],
$$




\section{ELA}

$$
\begin{aligned}
s \tilde{\Theta}_{1}-\tilde{\Phi}_{1}: & =\left[\begin{array}{ccc}
s \Theta_{11}-\Phi_{11} & s \tilde{\Theta}_{12}-\tilde{\Phi}_{12} & s \tilde{\Theta}_{13}-\tilde{\Phi}_{13} \\
s \Theta_{21}-\Phi_{21} & s \tilde{\Theta}_{22}-\tilde{\Phi}_{22} & s \tilde{\Theta}_{23}-\tilde{\Phi}_{23} \\
0 & s \tilde{\Theta}_{32}-\tilde{\Phi}_{32} & s \tilde{\Theta}_{33}-\tilde{\Phi}_{33} \\
0 & 0 & s \tilde{\Theta}_{43}-\tilde{\Phi}_{43}
\end{array}\right], \quad \tilde{\Psi}:=\left[\begin{array}{c}
\Psi_{1} \\
\Psi_{2} \\
\tilde{\Psi}_{3} \\
\tilde{\Psi}_{4}
\end{array}\right], \\
s \hat{\Theta}_{2}-\hat{\Phi}_{2}: & =\left[\begin{array}{ccc}
s \tilde{\Theta}_{43}-\tilde{\Phi}_{43} & s \tilde{\Theta}_{44}-\tilde{\Phi}_{44} \\
0 & s \Theta_{45}-\Phi_{45}
\end{array}\right], \\
s \tilde{\Theta}_{2}-\tilde{\Phi}_{2}: & =\left[\begin{array}{ccc}
s \tilde{\Theta}_{32}-\tilde{\Phi}_{32} & s \tilde{\Theta}_{33}-\tilde{\Phi}_{33} & s \tilde{\Theta}_{34}-\tilde{\Phi}_{34} \\
0 & s \tilde{\Theta}_{43}-\tilde{\Phi}_{43} & s \tilde{\Theta}_{44}-\tilde{\Phi}_{44} \\
0 & 0 & s \Theta_{45}-\Phi_{45}
\end{array}\right], \\
\tilde{\Pi} & :=\left[\begin{array}{ccc}
\tilde{C}_{2} & \tilde{C}_{3} & \tilde{C}_{4}
\end{array}\right] .
\end{aligned}
$$

Note that $\Theta_{33}$ is nonsingular, $\Psi_{4}$ is of full row rank, and $C_{2}$ is of full column rank, so $\left[\begin{array}{cc}\tilde{\Theta}_{43} & \tilde{\Psi}_{4}\end{array}\right]$ is of full row rank and $\left[\begin{array}{c}\tilde{\Theta}_{32} \\ \tilde{C}_{2}\end{array}\right]$ is of full column rank, i.e.,

$$
\operatorname{rank}\left[\begin{array}{cc}
\tilde{\Theta}_{43} & \tilde{\Psi}_{4}
\end{array}\right]=\left(\mu_{3}+\tau_{4}+k\right)-w, \quad \operatorname{rank}\left[\begin{array}{c}
\tilde{\Theta}_{32} \\
\tilde{C}_{2}
\end{array}\right]=v .
$$

Since the pencil $(\mathcal{E}, \mathcal{A})$ is regular and of index at most one, the same also holds for the pencils $\left(\hat{\Theta}_{1}, \hat{\Phi}_{1}\right)$ and $\left(\hat{\Theta}_{2}, \hat{\Phi}_{2}\right)$. Thus, Lemma 1.3 implies that

$$
\operatorname{rank}\left[\begin{array}{cc}
\hat{\Theta}_{1} & \hat{\Phi}_{1} S_{\infty}\left(\hat{\Theta}_{1}\right)
\end{array}\right]=\tau_{1}+\tau_{2}+w
$$

and

$$
\operatorname{rank}\left[\begin{array}{c}
\hat{\Theta}_{2} \\
T_{\infty}^{T}\left(\hat{\Theta}_{2}\right) \hat{\Phi}_{2}
\end{array}\right]=\left(\mu_{2}+\mu_{3}+k-v\right)+\mu_{4} .
$$

Therefore, using (38), we have that

$$
\operatorname{rank}\left[\begin{array}{ccc}
\tilde{\Theta}_{1} & \tilde{\Phi}_{1} S_{\infty}\left(\tilde{\Theta}_{1}\right) & \tilde{\Psi}
\end{array}\right]=\tau_{1}+\tau_{2}+\mu_{3}+\tau_{4}+k
$$

and

$$
\operatorname{rank}\left[\begin{array}{c}
\tilde{\Theta}_{2} \\
T_{\infty}^{T}\left(\tilde{\Theta}_{2}\right) \tilde{\Phi}_{2} \\
\tilde{\Pi}
\end{array}\right]=\mu_{2}+\mu_{3}+\mu_{4}+k
$$

or, in other words,

$$
\operatorname{rank}\left[\begin{array}{ccccc}
\tilde{E}_{1} & 0 & \tilde{A}_{1} S_{\infty}\left(\tilde{E}_{1}\right) & \tilde{B} & 0 \\
0 & E_{m} & 0 & 0 & I
\end{array}\right]=\tau_{1}+\tau_{2}+\mu_{3}+\tau_{4}+k
$$

and

$$
\operatorname{rank}\left[\begin{array}{cc}
\tilde{E}_{2} & 0 \\
0 & E_{m} \\
T_{\infty}^{T}\left(\tilde{E}_{2}\right) \tilde{A}_{2} & 0 \\
\tilde{C} & 0 \\
0 & I
\end{array}\right]=\mu_{2}+\mu_{3}+\mu_{4}+k
$$




\section{ELA}

i.e., conditions (32) and (33) follow.

To prove condition (31), note that $\Theta_{33}$ is nonsingular and $\tilde{\Theta}_{32}$ is of full row rank, so we have

$$
\begin{aligned}
\operatorname{rank}\left(\Theta_{33}\right) & +\operatorname{rank}\left(E_{m}\right)+\operatorname{rank}\left[\begin{array}{c}
\Theta_{44} \\
\Theta_{54}
\end{array}\right] \\
& =\operatorname{rank}\left[\begin{array}{ccc}
\Theta_{33} & 0 & \Theta_{34} \\
0 & 0 & \Theta_{44} \\
0 & E_{m} & 0 \\
0 & 0 & \Theta_{54}
\end{array}\right] \\
& =\operatorname{rank}\left[\begin{array}{ccc}
\tilde{\Theta}_{32} & \tilde{\Theta}_{33} & \tilde{\Theta}_{34} \\
0 & \tilde{\Theta}_{43} & \tilde{\Theta}_{44} \\
0 & 0 & \Theta_{54}
\end{array}\right] \\
& =\operatorname{rank}\left[\begin{array}{cc}
\tilde{\Theta}_{32} & \tilde{\Theta}_{33} \\
0 & \tilde{\Theta}_{43}
\end{array}\right]+\operatorname{rank}\left[\begin{array}{c}
T_{\infty}^{T}\left(\tilde{\Theta}_{43}\right) \tilde{\Theta}_{44} \\
\Theta_{54}
\end{array}\right] \\
& =\operatorname{rank}\left(\Theta_{33}\right)+\operatorname{rank}\left(E_{m}\right)+\operatorname{rank}\left[\begin{array}{c}
T_{\infty}^{T}\left(\tilde{\Theta}_{43}\right) \tilde{\Theta}_{44} \\
\Theta_{54}
\end{array}\right]
\end{aligned}
$$

and hence

$$
\operatorname{rank}\left[\begin{array}{c}
\Theta_{44} \\
\Theta_{54}
\end{array}\right]=\operatorname{rank}\left[\begin{array}{c}
T_{\infty}^{T}\left(\tilde{\Theta}_{43}\right) \tilde{\Theta}_{44} \\
\Theta_{54}
\end{array}\right] .
$$

Moreover, since $(\mathcal{E}, \mathcal{A})$ is regular and of index at most one, by Lemma 1.3 (d) we obtain that

$$
\begin{aligned}
& \operatorname{rank}(E)+\operatorname{rank}\left(E_{m}\right)=\operatorname{rank}\left[\begin{array}{cc}
\Theta_{11} & \tilde{\Theta}_{12} \\
\Theta_{21} & \tilde{\Theta}_{22} \\
0 & \tilde{\Theta}_{32}
\end{array}\right]+\operatorname{rank}\left[\begin{array}{cc}
\tilde{\Theta}_{43} & \tilde{\Theta}_{44} \\
0 & \Theta_{54}
\end{array}\right] \\
& =\operatorname{rank}\left[\begin{array}{ccc}
\Theta_{11} & \tilde{\Theta}_{12} & 0 \\
\Theta_{21} & \tilde{\Theta}_{22} & 0 \\
0 & \tilde{\Theta}_{32} & 0 \\
0 & 0 & \tilde{\Theta}_{43}
\end{array}\right]+\operatorname{rank}\left[\begin{array}{c}
T_{\infty}^{T}\left(\tilde{\Theta}_{43}\right) \tilde{\Theta}_{44} \\
\Theta_{54}
\end{array}\right] \\
& \leq \operatorname{rank}\left[\begin{array}{ccc}
\Theta_{11} & \tilde{\Theta}_{12} & \tilde{\Theta}_{13} \\
\Theta_{21} & \tilde{\Theta}_{22} & \tilde{\Theta}_{23} \\
0 & \tilde{\Theta}_{32} & \tilde{\Theta}_{33} \\
0 & 0 & \tilde{\Theta}_{43}
\end{array}\right]+\operatorname{rank}\left[\begin{array}{c}
\Theta_{44} \\
\Theta_{54}
\end{array}\right] \\
& =\operatorname{rank}\left[\begin{array}{cccc}
\Theta_{11} & \Theta_{12} & \Theta_{13} & 0 \\
\Theta_{21} & \Theta_{22} & \Theta_{23} & 0 \\
0 & 0 & \Theta_{33} & 0 \\
0 & 0 & 0 & 0 \\
0 & 0 & 0 & E_{m}
\end{array}\right]+\operatorname{rank}\left[\begin{array}{c}
\Theta_{44} \\
\Theta_{54}
\end{array}\right] \text {, }
\end{aligned}
$$


i.e., we have that

$$
\operatorname{rank}(E) \leq \mu_{3}+\operatorname{rank}\left[\begin{array}{cc}
\Theta_{11} & \Theta_{12} \\
\Theta_{21} & \Theta_{22}
\end{array}\right]+\operatorname{rank}\left[\begin{array}{c}
\Theta_{44} \\
\Theta_{54}
\end{array}\right]
$$

Since the other direction of this inequality is obvious, condition (31) follows. (d) follows from (b) and (c).

The following result gives sufficient conditions. In the course of the proof we explicitly construct the desired feedbacks.

THEOREM 3.2. Consider a system of the form (1) and assume that orthogonal matrices $P$ are given such that $P E Q, P A Q, P B, P G, C Q$, and $H Q$ are in the condensed form (9). Let $\tilde{E}_{1}, \tilde{E}_{2}, \tilde{A}_{1}, \tilde{A}_{2}, \tilde{B}, \tilde{C}, \Lambda_{i}, i=1, \ldots, 6, \Lambda_{r}, \Lambda_{t}, \Pi, \Xi_{j}$, $j=1,2,3$, be as in Corollary 2.3 .

(a) If the conditions (12)-(16) hold, then there exists a compensator of the form (2) such that the pencil of the closed-loop system (3) is regular and the disturbance decoupling relation (4) holds.

(b) If conditions (14) and (17)-(20) hold, then there exists a compensator of the form (2) such that the pencil of the closed-loop system (3) is regular, stable, and (4) holds.

(c) If conditions (14) and (21)-(25) hold, then there exists a compensator of the form (2) such that the pencil of the closed-loop system (3) is regular, of index at most one, and (4) holds.

(d) If conditions (14) and (17)-(25) hold, then there exists a compensator of the form (2) such that the pencil of the closed-loop system (3) is regular, stable, of index at most one, and (4) holds.

Proof. We prove Theorem 3.2 constructively. Since conditions (12) and (13) yield that

$$
\operatorname{rank}_{g}\left(s \Theta_{54}-\Phi_{54}\right)=\tau_{5}, \quad \operatorname{rank}_{g}\left[\begin{array}{c}
s \Theta_{11}-\Phi_{11} \\
s \Theta_{21}-\Phi_{22}
\end{array}\right]=\mu_{1}
$$

by computing the GUPTRI forms of $s \Theta_{54}-\Phi_{54}$ and $\left[\begin{array}{c}s \Theta_{11}-\Phi_{11} \\ s \Theta_{21}-\Phi_{21}\end{array}\right]$ and using that (26) holds, we can find orthogonal matrices $\tilde{U}_{1} \in \mathbb{R}^{\left(\tau_{1}+\tau_{2}\right) \times\left(\tau_{1}+\tau_{2}\right)}, \tilde{V}_{1} \in \mathbb{R}^{\mu_{1} \times \mu_{1}}$, $\tilde{V}_{2} \in \mathbb{R}^{\mu_{2} \times \mu_{2}}, \hat{U}_{4} \in \mathbb{R}^{\tau_{4} \times \tau_{4}}, \hat{U}_{5} \in \mathbb{R}^{\tau_{5} \times \tau_{5}}$, and $\hat{V}_{4} \in \mathbb{R}^{\mu_{4} \times \mu_{4}}$ such that

$$
\tilde{U}_{1}\left[\begin{array}{cc}
s \Theta_{11}-\Phi_{11} & s \Theta_{12}-\Phi_{12} \\
s \Theta_{21}-\Phi_{21} & s \Theta_{22}-\Phi_{22}
\end{array}\right]\left[\begin{array}{ll}
\tilde{V}_{1} & \\
& \tilde{V}_{2}
\end{array}\right]
$$

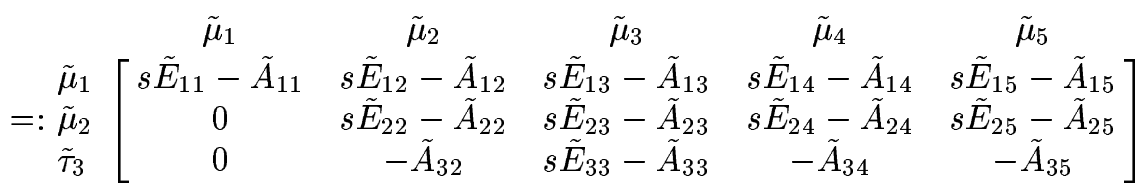




\section{ELA}

and

$$
\left[\begin{array}{cc}
\hat{U}_{4} & \\
& \hat{U}_{5}
\end{array}\right]\left[\begin{array}{c}
s \Theta_{44}-\Phi_{44} \\
s \Theta_{54}-\Phi_{54}
\end{array}\right] \hat{V}_{4}=\begin{array}{cccc}
\hat{\tau}_{4} & \hat{\tau}_{5} & \hat{\mu}_{6} \\
& \hat{\tau}_{6} \\
& \hat{\mu}_{5} \\
& \hat{\mu}_{6}
\end{array}\left[\begin{array}{ccc}
-\hat{A}_{44} & s \hat{E}_{45}-\hat{A}_{45} & s \hat{E}_{46}-\hat{A}_{46} \\
-\hat{A}_{54} & s \hat{E}_{55}-\hat{A}_{55} & s \hat{E}_{56}-\hat{A}_{56} \\
s \hat{E}_{64}-\hat{A}_{64} & s \hat{E}_{65}-\hat{A}_{65} & s \hat{E}_{66}-\hat{A}_{66} \\
-\hat{A}_{74} & s \hat{E}_{75}-\hat{A}_{75} & s \hat{E}_{76}-\hat{A}_{76} \\
0 & 0 & s \hat{E}_{86}-\hat{A}_{86}
\end{array}\right],
$$

where $\tilde{E}_{22}$ and $\hat{E}_{75}$ are nonsingular, $\tilde{E}_{33}$ is of full column rank, $\hat{E}_{64}$ is of full row rank, and $s \tilde{E}_{11}-\tilde{A}_{11}$ and $s \hat{E}_{86}-\hat{A}_{86}$ are nonsingular for any $s \in \mathbb{C}$. Furthermore,

$$
\begin{aligned}
\tilde{\tau}_{3} & =\left(\tau_{1}+\tau_{2}\right)-\left(\tilde{\mu}_{1}+\tilde{\mu}_{2}\right), \quad \tilde{\mu}_{5}=\tau_{4}+\tau_{5}-\mu_{4}, \\
\tilde{\mu}_{4} & =\mu_{2}-\tilde{\mu}_{3}-\tilde{\mu}_{5}, \quad \tilde{\mu}_{1}+\tilde{\mu}_{2}=\mu_{1}, \\
\hat{\mu}_{4} & =\mu_{4}-\hat{\mu}_{5}-\hat{\mu}_{6}, \quad \hat{\tau}_{4}=\left(\mu_{1}+\mu_{2}\right)-\left(\tau_{1}+\tau_{2}\right), \\
\hat{\tau}_{5} & =\tau_{4}-\hat{\tau}_{4}-\hat{\tau}_{6}, \quad \hat{\mu}_{5}+\hat{\mu}_{6}=\tau_{5} .
\end{aligned}
$$

Set

$$
\begin{gathered}
\hat{U}_{4}\left[\begin{array}{ll}
\Phi_{42} & \Phi_{43}
\end{array}\right]=: \begin{array}{cc}
\mu_{4} & \mu_{3} \\
\hat{\tau}_{5} \\
\hat{\tau}_{6}
\end{array}\left[\begin{array}{ll}
\hat{A}_{42} & \hat{A}_{43} \\
\hat{A}_{52} & \hat{A}_{53} \\
\hat{A}_{62} & \hat{A}_{63}
\end{array}\right], \\
{\left[\begin{array}{l}
\Phi_{32} \\
\Phi_{42}
\end{array}\right] \tilde{V}_{2}=: \begin{array}{lll}
\mu_{3} \\
\tau_{4}
\end{array}\left[\begin{array}{lll}
\tilde{\mu}_{3} & \tilde{\mu}_{4} & \tilde{\mu}_{5} \\
\tilde{A}_{53} & \tilde{A}_{54} & \tilde{A}_{45} \\
\tilde{A}_{55}
\end{array}\right],}
\end{gathered}
$$

and, furthermore,

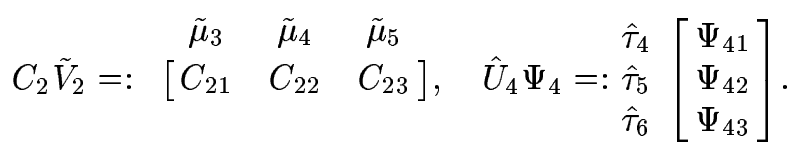

We partition $F^{b} \in \mathbb{R}^{m \times n}, F^{c} \in \mathbb{R}^{n \times p}$ analogously to the form (9) and set

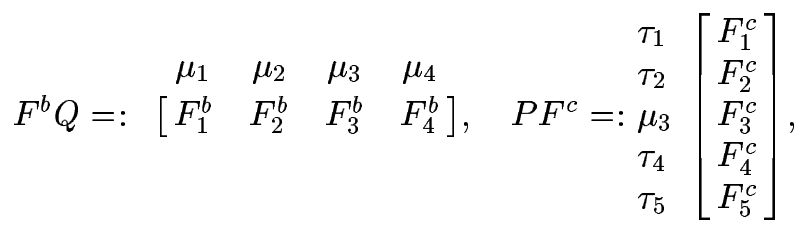

with

$$
\left.F_{4}^{b} \hat{V}_{4}=: \begin{array}{ccc}
\hat{\mu}_{4} & \hat{\mu}_{5} & \hat{\mu}_{6} \\
{\left[F_{41}^{b}\right.} & F_{42}^{b} & F_{43}^{b}
\end{array}\right], \quad \tilde{U}_{1}\left[\begin{array}{c}
F_{1}^{c} \\
F_{2}^{c}
\end{array}\right]=: \begin{aligned}
& \tilde{\mu}_{1} \\
& \tilde{\mu}_{2} \\
& \tilde{\tau}_{3}
\end{aligned}\left[\begin{array}{c}
F_{11}^{c} \\
F_{21}^{c} \\
F_{31}^{c}
\end{array}\right] .
$$


If $N$ is a solution of the linear system

$$
\Psi_{4} N=F_{4}^{c},
$$

then set

$$
\begin{aligned}
E_{m} & :=E, A_{m}:=A+B F^{b}+F^{c} C-B N C \\
L & :=B N-F^{c}, M:=F^{b}-N C .
\end{aligned}
$$

Then, for all $s \in \mathbb{C}$, we have

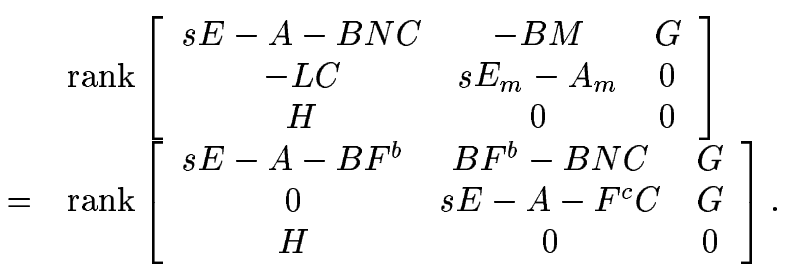

(a) Since conditions (27) and (28) hold, by Lemma 1.4 (a), we can compute $F_{1}^{b}, F_{2}^{b}$, $F_{3}^{b}, F_{3}^{c}, F_{4}^{c}$, and $F_{5}^{c}$ such that

$$
\begin{gathered}
{\left[\begin{array}{l}
\Psi_{42} \\
\Psi_{43}
\end{array}\right]\left[\begin{array}{lll}
F_{1}^{b} & F_{2}^{b} & F_{3}^{b}
\end{array}\right]+\left[\begin{array}{ccc}
0 & \hat{A}_{52} & \hat{A}_{53} \\
0 & \hat{A}_{62} & \hat{A}_{63}
\end{array}\right]=0} \\
{\left[\begin{array}{c}
F_{3}^{c} \\
F_{4}^{c} \\
F_{5}^{c}
\end{array}\right]\left[\begin{array}{ll}
C_{21} & C_{22}
\end{array}\right]+\left[\begin{array}{cc}
\tilde{A}_{43} & \tilde{A}_{44} \\
\tilde{A}_{53} & \tilde{A}_{54} \\
0 & 0
\end{array}\right]=0}
\end{gathered}
$$

and, for at least one $s \in \mathbb{C}$,

$$
\begin{aligned}
\operatorname{det}\left[\begin{array}{ccc}
s \Theta_{11}-\Phi_{11}-\Psi_{1} F_{1}^{b} & s \Theta_{12}-\Phi_{12}-\Psi_{1} F_{2}^{b} & s \Theta_{13}-\Phi_{13}-\Psi_{3} F_{3}^{b} \\
s \Theta_{21}-\Phi_{21}-\Psi_{2} F_{1}^{b} & s \Theta_{22}-\Phi_{22}-\Psi_{2} F_{2}^{b} & s \Theta_{23}-\Phi_{23}-\Psi_{2} F_{3}^{b} \\
-\Psi_{3} F_{1}^{b} & -\Phi_{32}-\Psi_{3} F_{2}^{b} & s \Theta_{33}-\Phi_{33}-\Psi_{3} F_{3}^{b} \\
-\Psi_{41} F_{1}^{b} & -\hat{A}_{42}-\Psi_{41} F_{2}^{b} & -\hat{A}_{43}-\Psi_{43} F_{3}^{b}
\end{array}\right] \neq 0, \\
\operatorname{det}\left[\begin{array}{ccc}
-\tilde{A}_{45}-F_{3}^{c} C_{23} & s \Theta_{33}-\Phi_{33}-F_{3}^{c} C_{3} & s \Theta_{34}-\Phi_{34}-F_{3}^{c} C_{4} \\
-\tilde{A}_{55}-F_{4}^{c} C_{23} & -\Phi_{43}-F_{4}^{c} C_{3} & s \Theta_{44}-\Phi_{44}-F_{4}^{c} C_{4} \\
-F_{5}^{c} C_{23} & -F_{5}^{c} C_{3} & s \Theta_{54}-\Phi_{54}-F_{5}^{c} C_{4}
\end{array}\right] \neq 0 .
\end{aligned}
$$

Moreover, conditions (12) and (13) hold, $\left[\begin{array}{l}\Psi_{42} \\ \Psi_{43}\end{array}\right]$ and $\left[\begin{array}{ll}C_{21} & C_{22}\end{array}\right]$ are of full row rank and full column rank, respectively, so there exists at least one $s \in \mathbb{C}$ such that

$$
\begin{array}{r}
\operatorname{rank}\left[\begin{array}{cccc}
-\hat{A}_{54} & s \hat{E}_{55}-\hat{A}_{55} & s \hat{E}_{56}-\hat{A}_{56} & \Psi_{42} \\
s \hat{E}_{64}-\hat{A}_{64} & s \hat{E}_{65}-\hat{A}_{65} & s \hat{E}_{66}-\hat{A}_{66} & \Psi_{43} \\
-\hat{A}_{74} & s \hat{E}_{75}-\hat{A}_{75} & s \hat{E}_{76}-\hat{A}_{76} & 0 \\
0 & 0 & s \hat{E}_{86}-\hat{A}_{86} & 0
\end{array}\right] \\
=\hat{\tau}_{5}+\hat{\tau}_{6}+\hat{\mu}_{5}+\hat{\mu}_{6} \\
\end{array}
$$


and

(48) $\operatorname{rank}\left[\begin{array}{cccc}s \tilde{E}_{11}-\tilde{A}_{11} & s \tilde{E}_{12}-\tilde{A}_{12} & s \tilde{E}_{13}-\tilde{A}_{13} & s \tilde{E}_{14}-\tilde{A}_{14} \\ 0 & s \tilde{E}_{22}-\tilde{A}_{22} & s \tilde{E}_{23}-\tilde{A}_{23} & s \tilde{E}_{24}-\tilde{A}_{24} \\ 0 & -\tilde{A}_{32} & s \tilde{E}_{33}-\tilde{A}_{33} & -\tilde{A}_{34} \\ 0 & 0 & C_{21} & C_{22}\end{array}\right]=\sum_{i=1}^{4} \tilde{\mu}_{i}$.

Hence, using Lemma 1.4 (a) again, we have matrices $F_{41}^{b}, F_{42}^{b}, F_{43}^{b}, F_{11}^{c}, F_{21}^{c}$, and $F_{31}^{c}$ such that, for at least one $s \in \mathbb{C}$, we have

$$
\operatorname{det}\left[\begin{array}{ccc}
-\hat{A}_{54}-\Psi_{42} F_{41}^{b} & s \hat{E}_{55}-\hat{A}_{55}-\Psi_{42} F_{42}^{b} & s \hat{E}_{56}-\hat{A}_{56}-\Psi_{42} F_{43}^{b} \\
s \hat{E}_{64}-\hat{A}_{64}-\Psi_{43} F_{41}^{b} & s \hat{E}_{65}-\hat{A}_{65}-\Psi_{43} F_{42}^{b} & s \hat{E}_{66}-\hat{A}_{66}-\Psi_{43} F_{43}^{b} \\
-\hat{A}_{74} & s \hat{E}_{75}-\hat{A}_{75} & s \hat{E}_{76}-\hat{A}_{76} \\
0 & 0 & s \hat{E}_{86}-\hat{A}_{86}
\end{array}\right]
$$

$(49)$

and

$$
\operatorname{det}\left[\begin{array}{cccc}
s \tilde{E}_{11}-\tilde{A}_{11} & s \tilde{E}_{12}-\tilde{A}_{12} & s \tilde{E}_{13}-\tilde{A}_{13}-F_{11}^{c} C_{21} & s \tilde{E}_{14}-\tilde{A}_{14}-F_{11}^{c} C_{22} \\
0 & s \tilde{E}_{22}-\tilde{A}_{22} & s \tilde{E}_{23}-\tilde{A}_{23}-F_{21}^{c} C_{21} & s \tilde{E}_{24}-\tilde{A}_{24}-F_{21}^{c} C_{22} \\
0 & -\tilde{A}_{32} & s \tilde{E}_{33}-\tilde{A}_{33}-F_{31}^{c} C_{21} & -\tilde{A}_{34}-F_{31}^{c} C_{22}
\end{array}\right]
$$

$\neq 0$.

$(50)$

Let $E_{m}, A_{m}, M$, and $L$ be defined by $(40)$ and $(41)$. Then $(\mathcal{E}, \mathcal{A})$ is regular. Furthermore, using (42) and Lemma 1.2, it is easy to verify that (4) holds.

(b) Since conditions (29) and (30) hold, by Lemma 1.4 (b) there exist matrices $F_{1}^{b}, F_{2}^{b}, F_{3}^{b}, F_{3}^{c}, F_{4}^{c}$, and $F_{5}^{c}$ such that (43) and (44) are satisfied and (45) and (46) hold for all $s \in \overline{\mathbb{C}}^{+}$. At the same time, conditions (17) and (18) give that (47) and (48) hold for all $s \in \overline{\mathbb{C}}^{+}$. Thus, by Lemma 1.4 (b), we can determine matrices $F_{41}^{b}$, $F_{42}^{b}, F_{43}^{b}, F_{11}^{c}, F_{21}^{c}$, and $F_{31}^{c}$ such that (49) and (50) hold for all $s \in \overline{\mathbb{C}}^{+}$. With $E_{m}$, $A_{m}, M$, and $L$ defined by (40) and (41), then $(\mathcal{E}, \mathcal{A})$ is regular, stable, and (4) holds.

(c) Since conditions (32) and (33) hold, by Lemma 1.4 (c), there exist matrices $F_{1}^{b}, F_{2}^{b}, F_{3}^{b}, F_{3}^{c}, F_{4}^{c}$, and $F_{5}^{c}$ such that (43) and (44) are satisfied, (45) and (46) hold for at least one $s \in \mathbb{C}$, and, furthermore,

$$
\begin{aligned}
\operatorname{deg} & \left(\operatorname{det}\left[\begin{array}{ccc}
s \Theta_{11}-\Phi_{11}-\Psi_{1} F_{1}^{b} & s \Theta_{12}-\Phi_{12}-\Psi_{1} F_{2}^{b} & s \Theta_{13}-\Phi_{13}-\Psi_{3} F_{3}^{b} \\
s \Theta_{21}-\Phi_{21}-\Psi_{2} F_{1}^{b} & s \Theta_{22}-\Phi_{22}-\Psi_{2} F_{2}^{b} & s \Theta_{23}-\Phi_{23}-\Psi_{2} F_{3}^{b} \\
-\Psi_{3} F_{1}^{b} & -\Phi_{32}-\Psi_{3} F_{2}^{b} & s \Theta_{33}-\Phi_{33}-\Psi_{3} F_{3}^{b} \\
-\Psi_{41} F_{1}^{b} & -\hat{A}_{42}-\Psi_{41} F_{2}^{b} & -\hat{A}_{43}-\Psi_{43} F_{3}^{b}
\end{array}\right]\right) \\
& =\operatorname{rank}\left[\begin{array}{ccc}
\Theta_{11} & \Theta_{12} & \Theta_{13} \\
\Theta_{21} & \Theta_{22} & \Theta_{23} \\
0 & 0 & \Theta_{33}
\end{array}\right],
\end{aligned}
$$


as well as

$$
\begin{aligned}
& \operatorname{deg}\left(\operatorname{det}\left[\begin{array}{ccc}
-\tilde{A}_{45}-F_{3}^{c} C_{23} & s \Theta_{33}-\Phi_{33}-F_{3}^{c} C_{3} & s \Theta_{34}-\Phi_{34}-F_{3}^{c} C_{4} \\
-\tilde{A}_{55}-F_{4}^{c} C_{23} & -\Phi_{43}-F_{4}^{c} C_{3} & s \Theta_{44}-\Phi_{44}-F_{4}^{c} C_{4} \\
-F_{5}^{c} C_{23} & -F_{5}^{c} C_{3} & s \Theta_{54}-\Phi_{54}-F_{5}^{c} C_{4}
\end{array}\right]\right) \\
& \quad=\operatorname{rank}\left[\begin{array}{cc}
\Theta_{33} & \Theta_{34} \\
0 & \Theta_{44} \\
0 & \Theta_{54}
\end{array}\right] .
\end{aligned}
$$

On the other hand, since conditions (21) and (22) hold, by Lemma 1.4 (c), there exist matrices $\hat{F}$ and $\tilde{F}$ such that the pencils $(E, A+B \hat{F})$ and $(E, A+\tilde{F} C)$ are regular and of index at most one. By applying Lemma $1.3(\mathrm{~d})$ to pencils $(E, A+B \hat{F})$ and $(E, A+\tilde{F} C)$, we obtain immediately that

$$
\begin{aligned}
& \hat{E}_{86}=0, \tilde{E}_{11}=0, \\
& \operatorname{rank}\left[\begin{array}{l}
\Theta_{44} \\
\Theta_{54}
\end{array}\right]=\operatorname{rank}\left[\begin{array}{ccc}
0 & \hat{E}_{55} & \hat{E}_{56} \\
\hat{E}_{64} & \hat{E}_{65} & \hat{E}_{66} \\
0 & \hat{E}_{75} & \hat{E}_{76}
\end{array}\right], \\
& \operatorname{rank}\left[\begin{array}{ll}
\Theta_{11} & \Theta_{12} \\
\Theta_{21} & \Theta_{22}
\end{array}\right]=\operatorname{rank}\left[\begin{array}{ccc}
\tilde{E}_{12} & \tilde{E}_{13} & \tilde{E}_{14} \\
\tilde{E}_{22} & \tilde{E}_{23} & \tilde{E}_{24} \\
0 & \tilde{E}_{33} & 0
\end{array}\right] .
\end{aligned}
$$

Note that $\hat{E}_{64}$ and $\left[\begin{array}{l}\Psi_{42} \\ \Psi_{43}\end{array}\right]$ are of full row rank, $\tilde{E}_{33}$ and $\left[\begin{array}{ll}C_{21} & C_{22}\end{array}\right]$ are of full column rank, and $\hat{A}_{86}, \hat{E}_{75}$, and, furthermore, $\tilde{A}_{11}$ and $\tilde{E}_{22}$ are nonsingular. Then, by Lemma 1.4 (c), there exist matrices $F_{4 i}^{b}, F_{i 1}^{c}, i=1,2,3$, such that (49) and (50) hold for at least one $s \in \mathbb{C}$ and, furthermore,

$$
\begin{aligned}
& \left.\operatorname{deg} \operatorname{det}\left[\begin{array}{ccc}
-\hat{A}_{54}-\Psi_{42} F_{41}^{b} & s \hat{E}_{55}-\hat{A}_{55}-\Psi_{42} F_{42}^{b} & s \hat{E}_{56}-\hat{A}_{56}-\Psi_{42} F_{43}^{b} \\
s \hat{E}_{64}-\hat{A}_{64}-\Psi_{43} F_{41}^{b} & s \hat{E}_{65}-\hat{A}_{65}-\Psi_{43} F_{42}^{b} & s \hat{E}_{66}-\hat{A}_{66}-\Psi_{43} F_{43}^{b} \\
-\hat{A}_{74} & s \hat{E}_{75}-\hat{A}_{75} & s \hat{E}_{76}-\hat{A}_{76} \\
0 & 0 & -\hat{A}_{86}
\end{array}\right]\right) \\
& =\operatorname{rank}\left[\begin{array}{ccc}
0 & \hat{E}_{55} & \hat{E}_{56} \\
\hat{E}_{64} & \hat{E}_{65} & \hat{E}_{66} \\
0 & \hat{E}_{75} & \hat{E}_{76}
\end{array}\right]=\operatorname{rank}\left[\begin{array}{c}
\Theta_{44} \\
\Theta_{45}
\end{array}\right],
\end{aligned}
$$

as well as

$$
\begin{gathered}
\operatorname{deg}\left(\operatorname{det}\left[\begin{array}{cccc}
-\tilde{A}_{11} & s \tilde{E}_{12}-\tilde{A}_{12} & s \tilde{E}_{13}-\tilde{A}_{13}-F_{11}^{c} C_{21} & s \tilde{E}_{14}-\tilde{A}_{14}-F_{11}^{c} C_{22} \\
0 & s \tilde{E}_{22}-\tilde{A}_{22} & s \tilde{E}_{23}-\tilde{A}_{23}-F_{21}^{c} C_{21} & s \tilde{E}_{24}-\tilde{A}_{24}-F_{21}^{c} C_{22} \\
0 & -\tilde{A}_{32} & s \tilde{E}_{33}-\tilde{A}_{33}-F_{31}^{c} C_{21} & -\tilde{A}_{34}-F_{31}^{c} C_{22}
\end{array}\right]\right) \\
=\operatorname{rank}\left[\begin{array}{ccc}
\tilde{E}_{12} & \tilde{E}_{13} & \tilde{E}_{14} \\
\tilde{E}_{22} & \tilde{E}_{23} & \tilde{E}_{24} \\
0 & \tilde{E}_{33} & 0
\end{array}\right]=\operatorname{rank}\left[\begin{array}{cc}
\Theta_{11} & \Theta_{12} \\
\Theta_{21} & \Theta_{22}
\end{array}\right] .
\end{gathered}
$$


Let $F^{b}$ and $F^{c}$ be determined by (39). Since we have (31), it is easy to verify that the pencils $\left(E, A+B F^{b}\right)$ and $\left(E, A+F^{c} C\right)$ are regular and of index at most one. With $E_{m}, A_{m}, M$, and $L$ defined by $(40)$ and $(41)$, we then have that $(\mathcal{E}, \mathcal{A})$ is regular and of index at most one and (4) holds.

(d) Since conditions (21), (22), (29), and (30) hold, by Lemma 1.4 (d), there exist matrices $F_{1}^{b}, F_{2}^{b}, F_{3}^{b}, F_{3}^{c}, F_{4}^{c}$, and $F_{5}^{c}$ such that (43), (44), (51), and (52) hold, and furthermore (45) and (46) hold for any $s \in \overline{\mathbb{C}}^{+}$. Similarly to parts (b) and (c), since conditions $(21),(22),(17)$, and (18) hold, by Lemma 1.4 (d) again, there exist matrices $F_{4 i}^{b}, F_{i 1}^{c}, i=1,2,3$, such that (53) and (54) hold and, furthermore, (49) and (50) are satisfied for all $s \in \overline{\mathbb{C}}^{+}$. Let $F^{b}$ and $F_{c}$ be defined by (39). Using (31), we have that the pencils $\left(E, A+B F^{b}\right)$ and $\left(E, A+F^{c} C\right)$ are regular, stable, and of index at most one. With $E_{m}, A_{m}, M$, and $L$ given by (40) and (41), we then have that $(\mathcal{E}, \mathcal{A})$ is regular, stable, and of index at most one and (4) holds. $\square$

REMARK 3.3. It should be noted that Theorem 3.1 is established under the assumption that $\left(E_{m}, A_{m}\right)$ is regular and of index at most one, while for $\left(E_{m}, A_{m}\right)$ in Theorem 3.2, we do not need this assumption. Apart from this difference, the conditions in these theorems present complete necessary and sufficient conditions.

In contrast to the conditions in Lemmas 1.2, 1.3, and 1.4, control theoretic interpretations of the necessary and sufficient conditions are difficult to give, since these conditions are intertwined to guarantee several properties at once.

4. Conclusions. We have presented necessary and sufficient conditions for the solution of the disturbance decoupling problem with the extra requirements that the resulting closed-loop system be regular, of index at most one, and stable.

The explicit construction of the feedbacks is described in the proof of Theorem 3.2 and is based on the condensed form (9), which can be computed via a numerically backward stable method as well as the explicit construction of regularizing feedbacks and stabilizing feedbacks described in $[4,15]$.

\section{REFERENCES}

[1] E. Anderson, Z. Bai, C. Bischof, J.W. Demmel, J. Dongarra, J. D. Croz, A. Greenbaum, S. Hammarling, A. McKenney, S. Ostrouchov, and D. Sorensen. LAPACK Users' Guide. Society for Industrial and Applied Mathematics, Philadelphia, 1992.

[2] M. Banaszuk, M. Kociecki, and K.M. Przyluski. The disturbance decoupling problem for implicit linear discrete-time systems. SIAM J. Control Optim., 28:1270-1293, 1990.

[3] A. Bunse-Gerstner, R. Byers, V. Mehrmann, and N.K. Nichols. Feedback design for regularizing descriptor systems. Linear Algebra Appl., 299:119-151, 1999.

[4] A. Bunse-Gerstner, V. Mehrmann, and N.K. Nichols. Regularization of descriptor systems by derivative and proportional state feedback. SIAM J. Matrix Anal. Appl., 13:46-67, 1992.

[5] A. Bunse-Gerstner, V. Mehrmann, and N.K. Nichols. Regularization of descriptor systems by output feedback. IEEE Trans. Automat. Control, AC-39:1742-1747, 1994.

[6] D. Chu and V. Mehrmann. Disturbance decoupled observer design for descriptor systems. Systems Control Lett., 38:37-48, 1999.

[7] D. Chu and V. Mehrmann. Disturbance decoupling for descriptor systems. SIAM J. Control Optim., 38:1830-1858, 2000.

[8] D. Chu, V. Mehrmann, and N.K. Nichols. Minimum norm regularization of descriptor systems by output feedback. Linear Algebra Appl., 296:39-77, 1999. 
[9] L. Dai. Singular Control Systems, volume 118 of Lecture Notes in Control and Information Sciences. Springer-Verlag, Berlin, 1989.

[10] J.W. Demmel and B. Kågström. The generalized Schur decomposition of an arbitrary pencil $A-\lambda B$ : Robust software with error bounds and applications. Part I: theory and algorithms. ACM Trans. Math. Software, 19:160-174, 1993.

[11] P. Van Dooren. The generalized eigenstructure problem in linear system theory. IEEE Trans. Automat. Control, AC-26:111-129, 1981.

[12] L.R. Fletcher and A. Asaraai. On disturbance decoupling in descriptor systems. SIAM J. Control Optim., 27:1319-1332, 1989.

[13] G.H. Golub and C.F. Van Loan. Matrix Computations, 3rd edition. The Johns Hopkins University Press, Baltimore, Maryland, 1996.

[14] G. Lebret. Structural solution of the disturbance decoupling problem for implicit linear discretetime systems. Circuits Systems Signal Process., 13:311-327, 1994.

[15] V. Mehrmann. The Autonomous Linear Quadratic Control Problem: Theory and Numerical Algorithms, volume 163 of Lecture Notes in Control and Information Sciences. SpringerVerlag, Heidelberg, 1991. 